\title{
Kaposi Sarcoma Herpes Virus (KSHV) infection inhibits macrophage formation and survival by counteracting Macrophage Colony-Stimulating Factor (M-CSF)-induced increase of Reactive Oxygen Species (ROS), c-Jun N-terminal kinase (JNK) phosphorylation and autophagy
}

\author{
Maria Saveria Gilardini Montani, Luca Falcinelli, Roberta Santarelli, Maria Anele Romeo, \\ Marisa Granato, Alberto Faggioni, Mara Cirone* \\ Department of Experimental Medicine, Sapienza University of Rome, Laboratory Affiliated to Istituto Pasteur Italia-Fondazione Cenci Bolognetti, Rome, Italy
}

A R T I C L E I N F O

\section{Keywords:}

Autophagy

KHSV

Monocytes

Reactive oxygen species

p62

\begin{abstract}
A B S T R A C T
Kaposi Sarcoma Herpes Virus (KSHV) is an oncovirus belonging to the human gammaherpesvirus family, able to infect several immune cell types including B cells, dendritic cells (DCs) and monocytes. In this study, we found that KSHV infection of monocytes counteracted the Reactive Oxygen Species (ROS) increase induced by Macrophage Colony-Stimulating Factor (M-CSF), prevented c-Jun N-terminal kinase (JNK) and B-cell lymphoma-2 (Bcl-2) phosphorylation and inhibited autophagy, leading to an impairment of cell survival and differentiation into macrophages. We also show that, to further dysregulate immune response in monocytes, KSHV reduced the production of pro-inflammatory cytokines such as Tumor necrosis factor alpha (TNF $\alpha$ ) while increased the release of the immune suppressive cytokine Interleukin-10 (IL-10). These results unveils new strategies put in place by KSHV to induce immune suppression and to persist into the infected host.
\end{abstract}

\section{Introduction}

KSHV is a gammaherpesvirus strongly involved in human cancer. Although its-associated malignancies mainly arise in immune compromised patients, the virus is able per se to induce immune suppression. Similarly to Epstein Barr virus (EBV) (Gilardini Montani et al., 2018a,b), KSHV up-regulated the inhibitory immune checkpoint Programmed death-ligand 1 (PD-L1) in monocytes (Host et al., 2017) and inhibited dendritic cell (DC) formation (Cirone et al., 2007; Santarelli et al., 2016). Moreover, KSHV can dysregulate the functions of differentiated DCs (Santarelli et al., 2014) and reduce Major Histocompatibility Complex (MHC) molecule surface expression (Brulois et al., 2014; Cirone et al., 2010). We have demonstrated that the dysregulation of autophagy is one of the underlying mechanisms leading to the KSHV-mediated impairment of DC formation (Santarelli et al., 2016) or function (Santarelli et al., 2014). This virus encodes for proteins able to inhibit the different phases of the autophagic process. Among those, vFlip that inhibits the first autophagic steps (Lee et al., 2009) and K7 reported to impair the fusion between autophagosomes and lysosomes
(Liang et al., 2013), step in which the autophagic cargo is degraded by the lysosomal proteases (Klionsky et al., 2016). Interestingly, the inhibition of the last autophagic phases has been reported to occur during KSHV and EBV replication, allowing the viruses to avoid their own degradation (Granato et al., 2014, 2015).

Autophagy has been reported to be essential for monocyte differentiation into macrophages (Zhang et al., 2012) and for the activation of c-Jun N-terminal kinase ( JNK) that may promote macrophage development and survival (Himes et al., 2006). The latter is also sustained by Reactive oxygen species (ROS) that also maintain activated several signaling pathways including JNK (Park et al., 2010). In this study, we investigated whether KSHV infection could interfere with the development of macrophages driven by M-CSF and interfere with its-induced production of ROS, JNK phosphorylation and autophagy.

Abbreviations: ACT, actin; ATG5, Autophagy-Related 5; CT, control; KSHV, Kaposi Sarcoma Herpes Virus; IL-10, Interleukin-10; NAC, N-acetylcysteine; clCASP3, cleaved caspase 3; ROS, reactive oxygen species; SP, SP600125; TNF, tumor necrosis factor

* Corresponding author at: Viale Regina Elena 324, 00161, Rome, Italy.

E-mail address: mara.cirone@uniroma1.it (M. Cirone). 
A

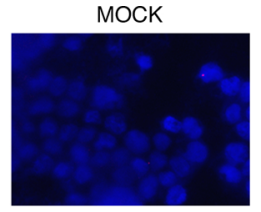

B
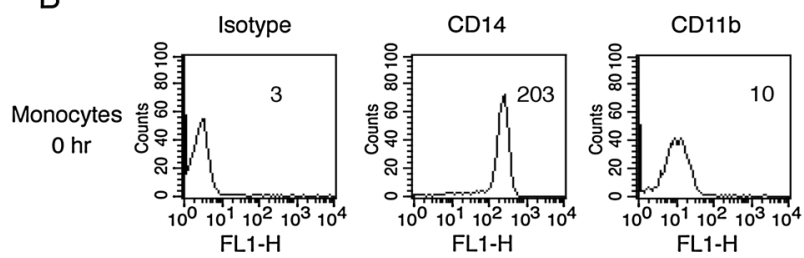

FL1-H

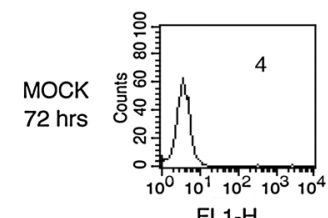

FL1-H

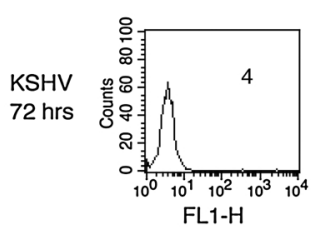

C
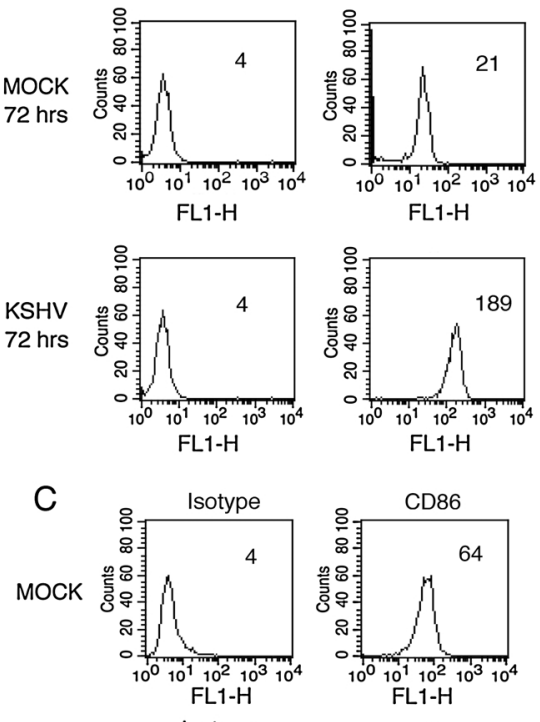

FL1-H
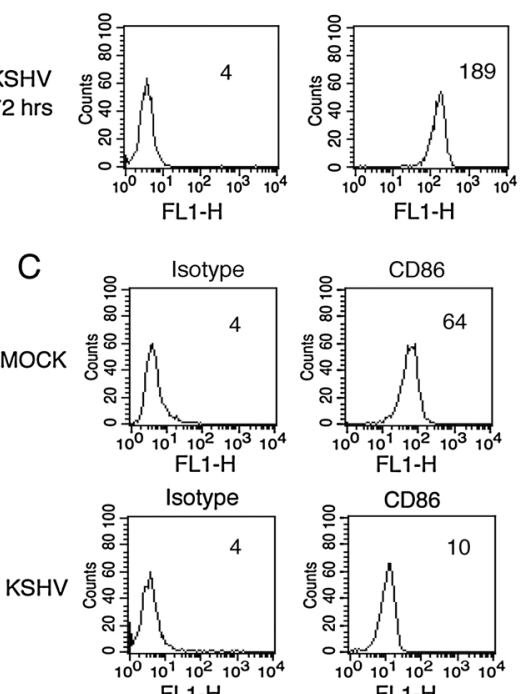

FL1-H
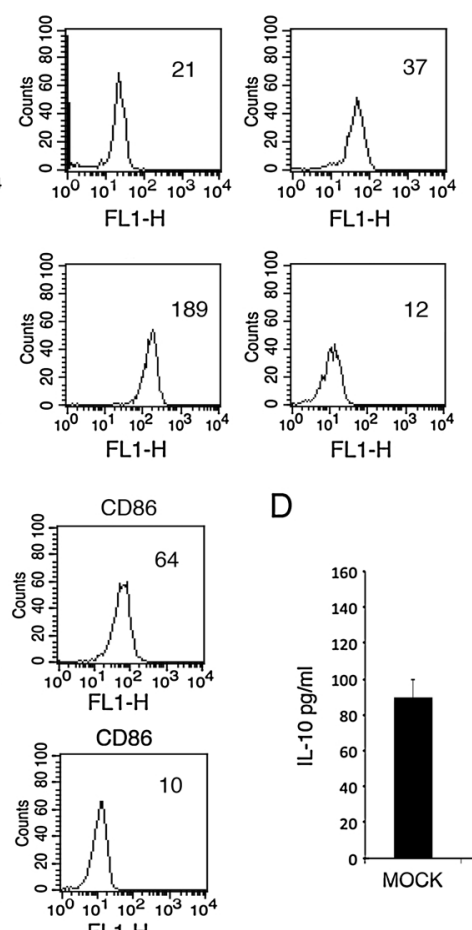

FL1-H

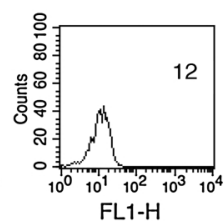

D

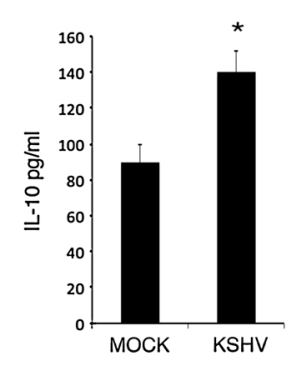

KSHV

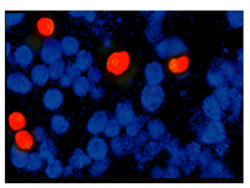

Fig. 1. KSHV infection impairs macrophage development and alters cytokine release.

A) K-bZIP expression in KSHV- and mock-infected monocytes as evaluated by IFA after $24 \mathrm{~h}$ of infection; B) FACS analysis of CD14, CD11b, CD86 and CD163 expression of KSHVand mock-infected monocytes cultured for $72 \mathrm{~h}$ in the presence of M-CSF. A representative experiment out of five is shown and the mean of fluorescence intensity is indicated C) FACS analysis of CD86 expression of KSHV- and mock-infected monocytes cultured for $72 \mathrm{~h}$ with M-CSF in the presence of LPS/IFNgamma. A representative experiment is shown and the mean of fluorescence intensity is indicated. D) and E) Elisa assay showing IL-10 and TNF alpha release by KSHV- and mock-infected monocytes cultured for 72 hs. Mean plus SD of three different experiments is reported. * $\mathrm{p}$ value $<0.05$.

\section{Material and methods}

\subsection{Monocytes isolation and KSHV infection}

Monocytes isolated from human peripheral blood mononuclear cells (PBMCs) of healthy donors as previously described (Gilardini Montani et al., 2018a,b) were infected with KSHV obtained as previously described (Cirone et al., 2007; Santarelli et al., 2016). Monocytes were infected at a multiplicity of infection (MOI) of 10 genome equivalents/ cell for $1 \mathrm{~h}$ at $37 \circ \mathrm{C}$ and then cultured for additional $72 \mathrm{hs}$ in complete medium. Differentiation into macrophages was performed by adding every two days $50 \mathrm{ng} / \mathrm{ml}$ M-CSF (Miltenyi Biotec, 130-096 and 130093) to the cultures. In some experiments, LPS/IFNgamma (100 ng/ml and $20 \mathrm{ng} / \mathrm{ml}$, respectively) was added to the culture for $24 \mathrm{~h}$ to promote cell activation.

\subsection{Indirect immunofluorescence assay (IFA)}

To detect KSHV infection, cells were applied onto multispot microscope slides (Thermo Scientific), air-dried, fixed with $2 \%$ paraformaldehyde (Electron Microscopy Science), washed three times in PBS and then permeabilized with $0.2 \%$ Triton X-100 (Sigma Aldrich)/PBS for $5 \mathrm{~min}$ at RT, as previously described (Santarelli et al., 2016). They were incubated with a primary monoclonal antibody against Kb-ZIP
(Santa Cruz Biotechnology, sc-69797), for $1 \mathrm{~h}$ at RT, and with a CyTM3conjugated sheep-anti-mouse secondary antibody for $30 \mathrm{~min}$ at RT (Jackson Imm. Res., 515-165-062). Cells were also stained with DAPI (4',6'-diamidino-2-phenylindole) $(1 \mu \mathrm{g} / \mathrm{ml})$ (Sigma Aldrich) and slides were observed by fluorescence microscope using Apotome Axio Observer Z1 inverted microscope (Zeiss, Oberkochen, Germany), equipped with an AxioCam MRM Rev. 3 at 40 x magnification.

\subsection{Immunofluorescence staining and FACS analysis}

After $72 \mathrm{~h}$, uninfected or KSHV-infected monocytes were stained with antibodies against CD14 (Miltenyi Biotec, 130-080-701), CD11b (Abcam, 34216), CD86 (Miltenyi Biotec, 130-094-877), CD163 (Santa Cruz Biotechnology, sc20066) and isotype control antibody (Miltenyi Biotec, 130-095-897) and analyzed by FACS Calibur flow cytometer (BD Transduction Laboratories), using CELLQuest software (BD Biosciences) (Gilardini Montani et al., 2016). For each analysis 10.000 events were recorded.

\subsection{Measurement of intracellular Reactive oxygen species production}

To measure ROS production, the $2^{\prime}, 7^{\prime}$-dichlorofluorescein diacetate (DCFDA) (Molecular Probes, CA) was used. DCFDA is a fluorogenic dye that, after diffusion into the cell, is oxidized by ROS into $2^{\prime}, 7^{\prime}$ - 
A
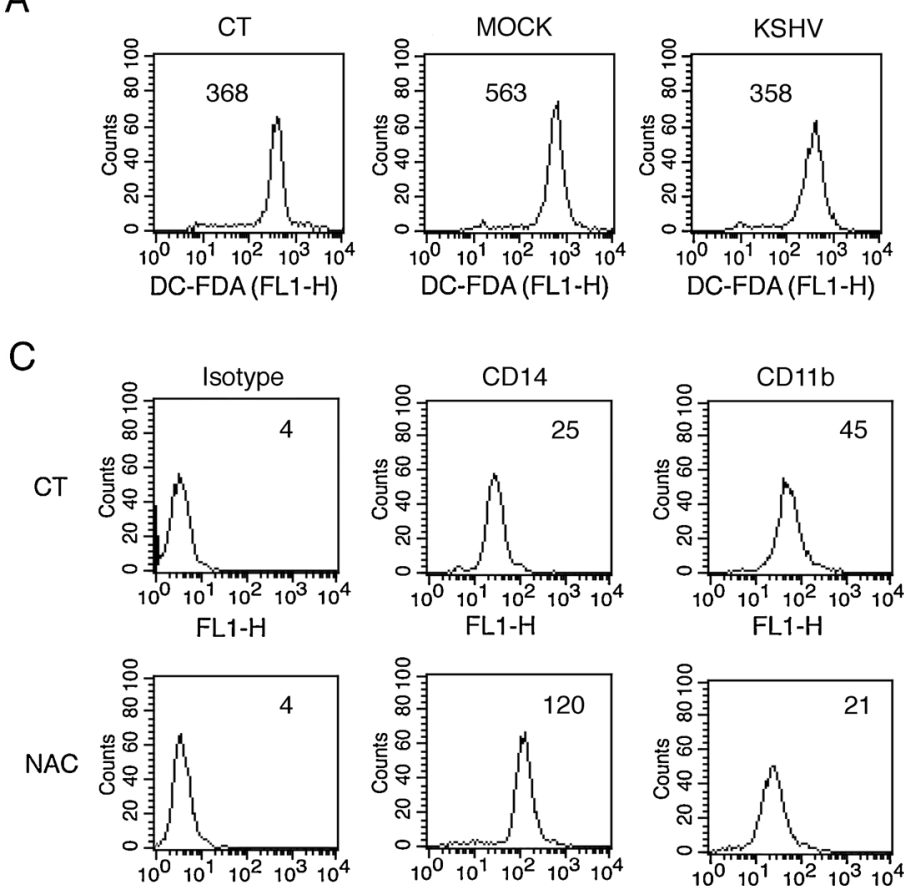

FL1-H

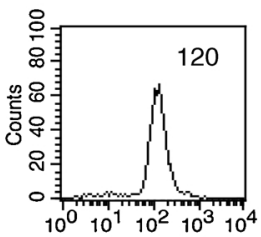

FL1-H
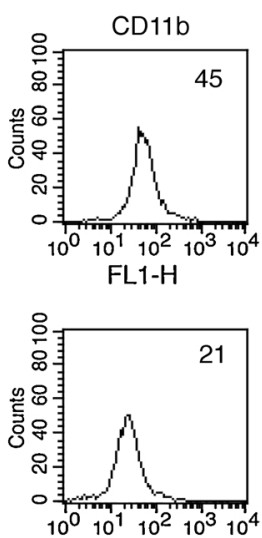

FL1-H
B
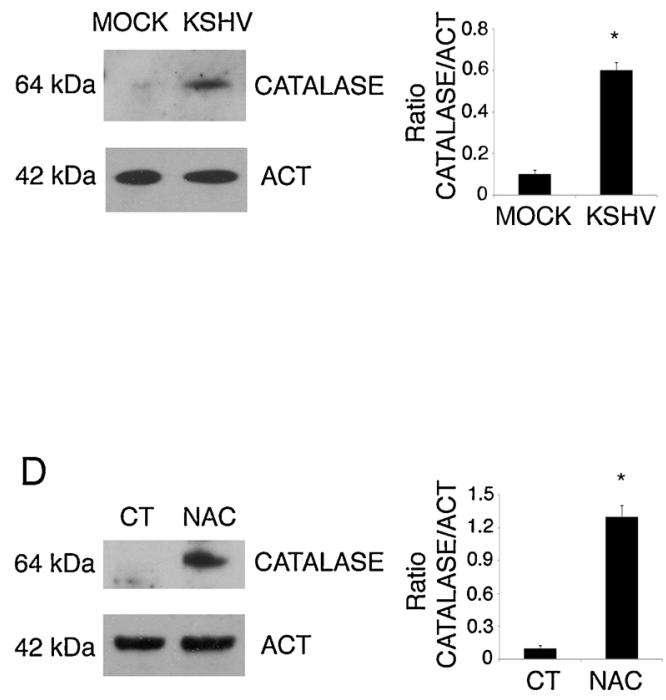

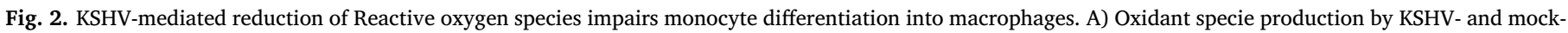

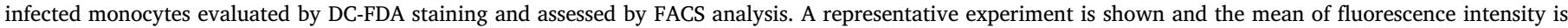

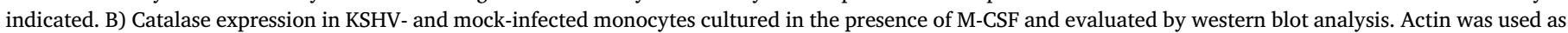

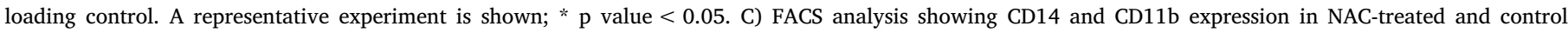

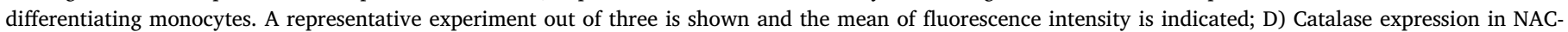

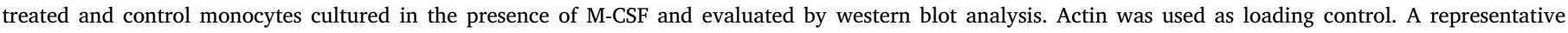
experiment out of three is shown. Histograms represent the mean plus S.D. of the densitometric analysis of the ratio of catalase/actin. * $\mathrm{p}$ value $<0.05$.

dichlorofluorescein (DCF), a highly fluorescent compound which can be detected by fluorescence spectroscopy. $72 \mathrm{~h}$ post infection, $5 \times 10^{5}$ KSHV-infected or uninfected CD14 ${ }^{+}$cells were incubated with $10 \mu \mathrm{M}$ DCFDA for $15 \mathrm{~min}$ in PBS at $37^{\circ} \mathrm{C}$. Then, cells were washed and analyzed in FL-1 by a FACS Calibur flow cytometer (BD Transduction Laboratories), using CELLQuest software (BD Biosciences) (Gilardini Montani et al., 2016). For each analysis 10.000 events were recorded.

\subsection{Cell viability}

After 24, 48 and $72 \mathrm{~h}$ of culture with $50 \mathrm{ng} / \mathrm{ml} \mathrm{M}$-CSF, a trypan blue (Euroclone) exclusion assay was performed to assess cell viability. In some experiments, monocytes were pretreated with $5 \mathrm{mM}$ N-Acetyl-Lcysteine (NAC) (Sigma Aldrich) or $10 \square \mathrm{M}$ SP600125 (JNK inhibitor) (Santa Cruz Biotechnology). Live cells were counted by light microscopy using a Neubauer hemocytometer. The experiments were performed in triplicate and repeated al least three times.

\subsection{Western blot analysis}

$1 \times 10^{6}$ uninfected or KSHV-infected cells were lysed, subjected to electrophoresis and transferred to nitrocellulose membranes, as previously described (Gilardini Montani et al., 2018a). Membranes were blocked in PBS- $0.1 \%$ Tween 20 solution containing 3\% BSA, probed with specific antibodies and developed using ECL Blotting Substrate (Advansta). The following antibodies were used: rabbit polyclonal antiPARP1 (1:500; Cell Signaling Technology, 9542), mouse monoclonal anti-catalase (1:100; Santa Cruz Biotechnology, sc-271803), rabbit polyclonal anti-p-JNK (1:300; Cell Signaling, 4668), mouse monoclonal anti-JNK (1:100; Santa Cruz Biotechnology sc-7345), mouse monoclonal anti-p-Bcl2 (1:100; Santa Cruz Biotechnology sc-293128), mouse monoclonal anti-Bcl2 (1:100; Santa Cruz Biotechnology sc-7382), rabbit polyclonal anti-LC3B (1:1000; Novus Biologicals, NB1002220SS), mouse monoclonal anti-SQSTM1 (1:500; BD Transduction Laboratories, 610883), rabbit polyclonal anti-ATG5 (1:500; Cell Signaling Technology, 2630), and anti-beta actin (1:10000; Sigma Aldrich, A5441). Goat anti-mouse IgG-HRP and anti-rabbit IgG-HRP (1:10.000 Santa Cruz Biotechnology Inc) were used as secondary antibodies.

\subsection{Knockdown by small interfering RNA (siRNA)}

ATG5 knockdown was performed by specific siRNA transfection (Santa Cruz Biotechnology, sc-41445) using HiPerFect Transfection Reagent (Qiagen, 301704) as previously described (Gilardini Montani et al., 2018b). Briefly, the transfection of $\mathrm{CD} 14^{+}$monocytes was done the same day, in which they were freshly isolated from blood and resuspended in warm complete RPMI at a cell density of $1.5 \times 10^{6}$ cells/ $\mathrm{ml}$ in $1 \mathrm{ml} /$ well in a 12-well plate format without antibiotics. A mix composed by $100 \mathrm{nM}$ ATG 5 siRNA with $15 \mu \mathrm{l}$ of HiPerFect Transfection Reagent was prepared and let react for $15 \mathrm{~min} \mathrm{RT}$, and then added to the cells. Control siRNA-A (Santa Cruz Biotechnology, sc-37007) was used as a scrambled control. After transfection, monocytes were cultured for additional $72 \mathrm{~h}$, in complete medium.

\subsection{Chemiluminescent immunometric assay}

After $72 \mathrm{~h}$ of culture, supernatants from KSHV-infected and mock control monocytes were collected and Interleukin-10 and TNF-alpha were measured by an automated solid-phase chemiluminescent immunometric assay performed by Immulite 1000 Analyzer (Siemens) using corresponding kits (IMMULITE ${ }^{\bullet}$ TNF-a and IMMULITE ${ }^{\bullet}$ IL-10, Diagnostic Products Corporation, Los Angeles, USA) according to the manufacture's instructions. 
A
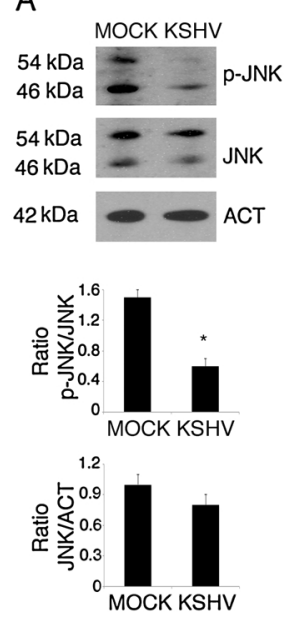

C
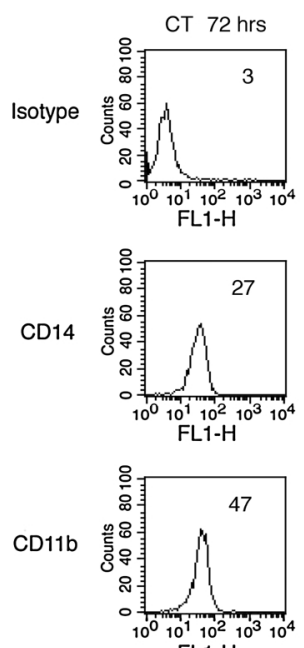

FL1-H
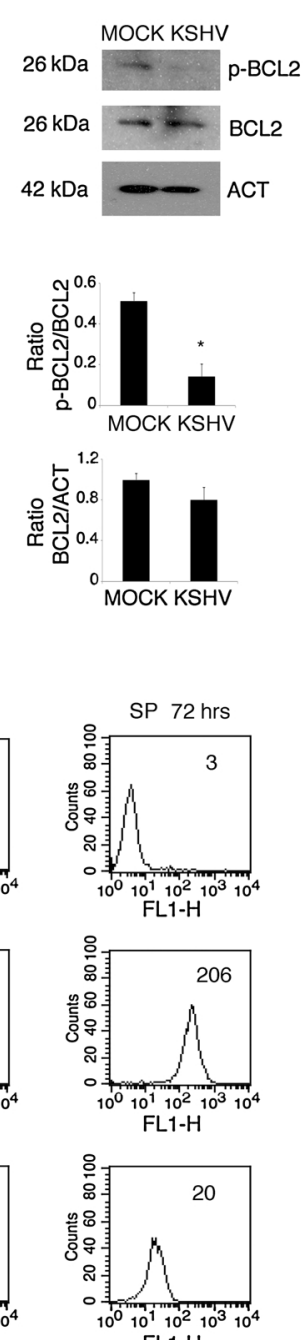

FL1-H
B
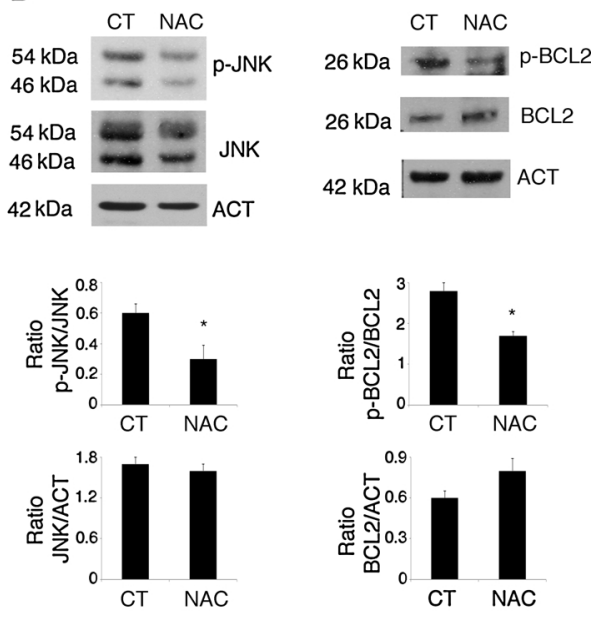

$\mathrm{D}$

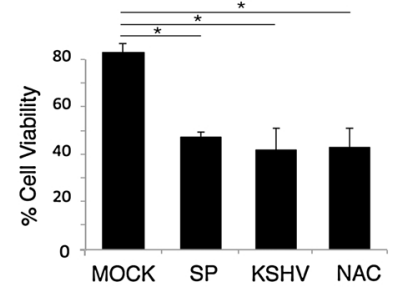

$\mathrm{E}$
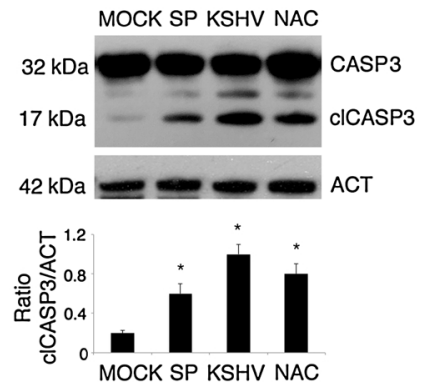

Fig. 3. The reduction of Reactive oxygen species by KSHV or NAC led to the de-phosphorylation of JNK required for macrophage formation and monocyte survival.

A) JNK and Bcl-2 phosphorylation in KSHVand mock-infected differentiating monocytes, as evaluated by western blot analysis; B) JNK and $\mathrm{Bcl}-2$ phosphorylation in NAC-treated and control differentiating monocytes as evaluated by western blot analysis. Actin was used as loading control and a representative experiment is shown; Histograms represent the mean plus S.D. of the densitometric analysis of the ratio of p-JNK/JNK, JNK/actin. p-BCL2/BCL2 and BCL2/actin; * p value $<0.05$. C) FACS analysis showing CD14 or CD11b expression of differentiating monocytes cultured in the presence or in the absence of SP 200125 JNK specific inhibitor. A representative experiments is shown and the mean of fluorescence intensity is indicated. D) Cell viability of mock or KSHV-infected or SP- or NAC-treated monocytes cultured in the presence of M-CSF, as evaluated by trypan blue exclusion assay. Mean plus SD of three independent experiments is reported. * p value $<0.05$; E) Caspase 3 cleavage of mock- of KSHV-infected or SP- or NAC-treated differentiating monocytes, as evaluated by western blot analysis. Actin was used as loading control. A representative experiments is shown. Histograms represent the mean plus S.D. of the densitometric analysis of the ratio of cleaved caspase $3 /$ actin. * p value $<0.05$.

\subsection{Densitometric analysis}

The quantification of proteins bands was performed by densitometric analysis using the Image $J$ software, which was downloaded from NIH web site (http://imagej.nih.gov).

\subsection{Statistical analysis}

Data are represented by the mean \pm standard deviation (SD) of at least three independent experiments and two-tailed Student's $t$-test was used for statistical significance of the differences between treatment groups. Difference was considered statistically significant when p-value was $\leq 0.05$.

\section{Results}

\subsection{KSHV infection reduces $M$-CSF-driven monocyte differentiation into} macrophages, prevents LPS/IFNgamma-induced activation and alters theirmediated cytokine release

Primary human monocytes were exposed to KSHV and, after $24 \mathrm{~h}$, infection was assessed by IFA. We found that infected monocytes expressed K-bZIP early lytic antigen (Fig. 1A), in agreement with the knowledge that the virus replicates in the early phases of infection (Purushothaman et al., 2016). Subsequently, KSHV- and mock-infected cells were cultured in the presence of M-CSF to induce their differentiation into macrophages. After $72 \mathrm{~h}$, KSHV-infected cells retained more CD14 monocyte marker and expressed lower level of CD11b, CD86 and CD163 in comparison with the mock-treated control cells (Fig. 1B), suggesting that viral infection interfered with the process of macrophage formation. Similar results were obtained when mock- or virus-infected monocytes were differentiated in macrophages in the presence of GM-CSF (data not shown). We then investigated the effect of viral infection on monocyte activation promoted by LPS/IFN gamma. As shown in Fig. 1C, KSHV counteracted CD86 up-regulation induced by LPS/INF gamma, suggesting that virus interfered with the activation of cells that could be detrimental for its survival. To investigate whether KSHV could also affect cytokine production in differentiating monocytes, pro-inflammatory and anti-inflammatory cytokine production was assessed in the supernatant of infected and control cells. We found that KSHV-infected monocytes produced a reduced amount of TNF alpha while increased IL-10 production (Fig. 1D and E). All together these results suggest that KSHV is able to reduce macrophage formation, prevent their activation and skew the cytokine release towards an immune suppressive profile, leading to an impairment of immune response.

\subsection{KSHV-infection reduces Reactive oxygen species (ROS) to impair macrophage formation}

Searching for the molecular mechanism/s that could underlie the impairment of monocyte to macrophage differentiation by KSHV, we 

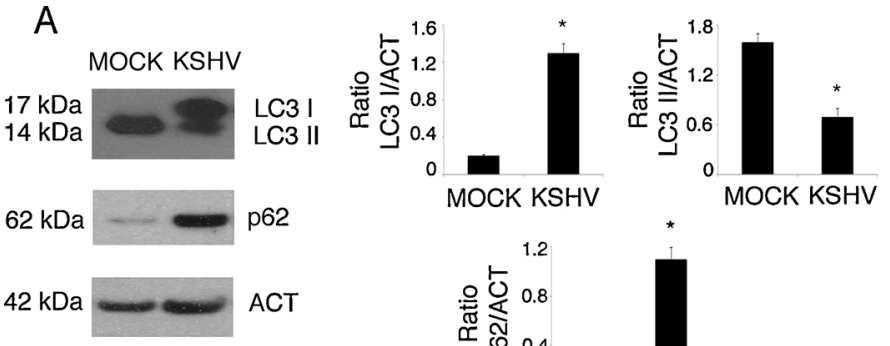

B

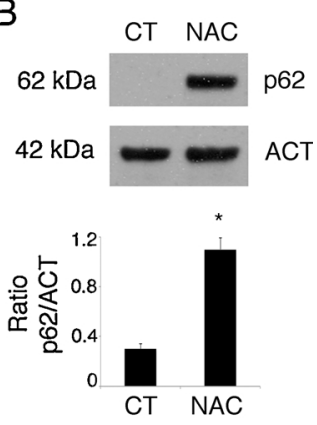

C
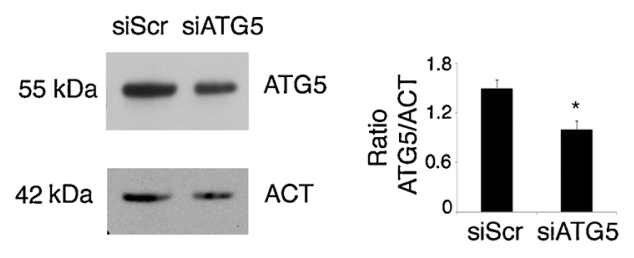

D
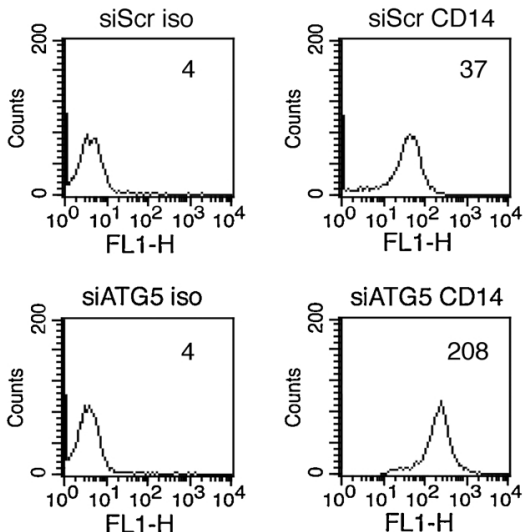

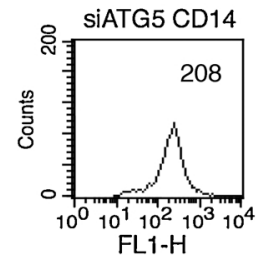

Fig. 4. KSHV-mediated reduction of autophagy contributes to the impairment of monocyte differentiation.

A) LC3I/II and p62 expression in KSHV- or mock-infected differentiating monocytes as evaluated by western blot analysis. Actin was used as loading control. A representative experiments is shown. Histograms represent the mean plus S.D. of the densitometric analysis of the ratio of LC3I/actin, LC3II/actin, p62/actin; * p value $<0.05$. B) p62 expression in NACtreated and control differentiating monocytes as evaluated by western blot analysis. Actin was used as loading control. A representative experiments is shown. Histograms represent the mean plus S.D. of the densitometric analysis of the ratio of p62/actin; * $\mathrm{p}$ value $<0.05$. C) ATG5 silencing assessed by western blot analysis. Actin was used as loading control. Histograms represent the mean plus S.D. of the densitometric analysis of the ratio of ATG5/ actin; * p value $<0.05$. D) Monocyte differentiation in ATG5 silenced or scramble-treated cells cultured in the presence of M-CSF, as evaluated by FACS analysis. A representative experiments is shown and the mean of fluorescence intensity is indicated. found that intracellular ROS, measured by DC-FDA increased by M-CSF, were reduced in virus-infected cells in comparison to the mock-infected control (Fig. 2A). As the ROS level is the result of the balance between their production and scavenging, mediated by the anti-oxidant enzymes, we investigated whether the latters could up-regulated and lead to the ROS reduction by KSHV infection. We found that the expression level of catalase, one of the most important enzymes involved in the anti-oxidant response, was increased by viral infection (Fig. 2B). This result indicates that KSHV by up-regulating catalase altered the balance between ROS production and scavenging leading to their reduction. Interestingly, it has been reported that ROS play an important role in the differentiating process of monocytes (Zhang et al., 2013) and another study has shown that the addition of catalase could prevent the differentiating process of THP1 human monocytoid cells (Yamamoto et al., 2009). All together these data suggest that reduction of ROS, likely mediated by the increase of catalase expression, could contribute to the KSHV-mediated impairment of macrophage formation. This hypothesis was supported by the findings that the ROS scavenger NAC was able to mimic the effects of KSHV infection in impairing macrophage formation (Fig. 2C), as it also increased catalase expression (Fig. 2D).

\subsection{The reduction of ROS reduces the phosphorylation of JNK required for monocyte differentiation and survival}

Among other pathways, ROS may induce the activation of JNK that in turn may induce the phosphorylation of Bcl-2. Thus, we next explored the status of JNK and Bcl2 phosphorylation in KSHV and in mock-infected monocytes undergoing M-CSF-treatment. As shown in Fig. 3A, the phosphorylation of both molecules was reduced in KSHVinfection in differentiating monocytes, in correlation with the reduction of ROS mediated by the virus. We then found that the ROS scavenger NAC mimicked the effects of KSHV in reducing JNK and Bcl-2 phosphorylation (Fig. 3B), confirming the role of ROS in maintaining this pathway activated. To evaluate the role of JNK de-phosphorylation in monocyte differentiation induced by M-CSF, we inhibited it by using
SP600125 and found that it led to a reduction of macrophage formation (Fig. 3C). Interestingly, besides monocyte differentiation, KSHV, SP600125 and NAC reduced cell survival (Fig. 3D) and increased caspase3 cleavage (Fig. 3E), in agreement to previous findings indicating that JNK was required for macrophage development and survival (Himes et al., 2006) and that also the ROS were involved in the latter effect (Bhatt et al., 2002).

\subsection{KSHV reduced autophagy contributing to the inhibition of macrophage development}

It has been reported that ROS represent one of the main stimuli able to activate autophagy (Azad et al., 2009; Filomeni et al., 2015). Also JNK and Bcl-2 phosphorylation that may be induced by ROS can promote the autophagic process (Zhong et al., 2017). Therefore, we investigated whether ROS reduction by KSHV could inhibit autophagy in differentiating monocytes. As shown in Fig. 4A, LC3II decreased and p62 accumulated in the virus-infected cells in comparison to the control cells, indicating that autophagy was reduced by viral infection. Similarly to KSHV infection, NAC also reduced autophagy in differentiating monocytes (Fig. 4B), indicating that ROS were involved in the activation of such process. According to the findings that autophagy was required for monocyte differentiation into macrophages (Zhang et al., 2012), we then found that the silencing of ATG5 (Fig. 4C) reduced the macrophage formation (Fig. 4D). These results suggest that the reduction of autophagy contributed to the impairment of monocyte differentiation mediated by KSHV.

\section{Discussion}

This study indicates that KSHV counteracted the M-CSF driven monocytes to macrophage differentiation and that the first ring of the chain of events leading to this effect was the reduction of ROS, whose production was induced by M-CSF. ROS, besides cell differentiation, were involved in monocyte survival, as indicated by the finding that the 
ROS scavenger NAC, that mimicked the effects induced by KSHV, impaired both cell survival and differentiation of monocytes. Interestingly, KSHV up-regulated catalase, one the most important enzymes mediating the anti-oxidant response and similar effects were observed by using NAC, according to previous findings (Zukowski et al., 2018). The level of ROS is tightly regulated by the equilibrium between their production and scavenging and, depending on their increase or decrease, several signaling pathways may result activated or inhibited. In this study, we found that the reduction of ROS induced by KSHV decreased the phosphorylation of JNK and Bcl-2. Interestingly, JNK phosphorylation has been previously reported to promote macrophage development (Himes et al., 2006), therefore its de-phosphorylation by KSHV could contribute to the viral-mediated impairment of monocyte differentiation. Another pathway known to be essential for macrophage formation and activated by the JNK-Bcl2 axis is autophagy that was also impaired by KSHV infection. ROS is one of the main stimuli that induces autophagy, thus their reduction may contribute to autophagy inhibition by KSHV. Of note, autophagy contributes to the direct microbial elimination and to viral antigen presentation (Santarelli et al., 2015), therefore the reduction of autophagy may have a variety of immune suppressive effects and represents a common strategy adopted by viruses to counteract their own elimination and persist in the infected host (Gilardini Montani et al., 2018b; Granato et al., 2016). Furthermore, as observed in infected DCs (Santarelli et al., 2014), KSHV dysregulated the cytokine release during M-CSF-driven monocyte differentiation. Indeed, it increased the release of the anti-inflammatory cytokine IL-10 and decreased the production of the pro-inflammatory cytokine TNF alpha, skewing cytokine release toward an immune suppressive profile. Interestingly, TNF alpha has been reported to increase intracellular ROS level (Woo et al., 2000) and to reduce catalase expression (Lupertz et al., 2008). Among the immune suppressive effects induced by KSHV, it also counteracted CD86 up-regulation in monocytes undergoing TLR stimulation with LPS/IFN gamma, suggesting that the virus can adopt several strategies to avoid immune recognition.

\section{Funding}

This work was supported by grants from Istituto Pasteur ItaliaFondazione Cenci Bolognetti.

\section{Acknowledgements}

We thank Prof. G. Girelli (Blood bank, Sapienza University of Rome) and Dr G. Mandarello and Dr R. Gasbarri (Immunohematology and Transfusion Service, Belcolle Hospital, Viterbo) for providing buffy coats.

\section{References}

Azad, M.B., Chen, Y., Gibson, S.B., 2009. Regulation of autophagy by reactive oxygen species (ROS): implications for cancer progression and treatment. Antioxid. Redox Signal. 11 (4), 777-790.

Bhatt, N.Y., Kelley, T.W., Khramtsov, V.V., Wang, Y., Lam, G.K., Clanton, T.L., Marsh, C.B., 2002. Macrophage-colony-stimulating factor-induced activation of extracellular-regulated kinase involves phosphatidylinositol 3-kinase and reactive oxygen species in human monocytes. J. Immunol. 169 (11), 6427-6434.

Brulois, K., Toth, Z., Wong, L.Y., Feng, P., Gao, S.J., Ensser, A., Jung, J.U., 2014. Kaposi's sarcoma-associated herpesvirus K3 and K5 ubiquitin E3 ligases have stage-specific immune evasion roles during lytic replication. J. Virol. 88 (16), 9335-9349.

Cirone, M., Di Renzo, L., Trivedi, P., Lucania, G., Borgia, G., Frati, L., Faggioni, A., 2010. Dendritic cell differentiation blocked by primary effusion lymphoma-released factors is partially restored by inhibition of P38 MAPK. Int. J. Immunopathol. Pharmacol. 23 (4), 1079-1086.

Cirone, M., Lucania, G., Bergamo, P., Trivedi, P., Frati, L., Faggioni, A., 2007. Human herpesvirus 8 (HHV-8) inhibits monocyte differentiation into dendritic cells and impairs their immunostimulatory activity. Immunol. Lett. 113 (1), 40-46.

Filomeni, G., De Zio, D., Cecconi, F., 2015. Oxidative stress and autophagy: the clash between damage and metabolic needs. Cell Death Differ. 22 (3), 377-388.

Gilardini Montani, M.S., Granato, M., Cuomo, L., Valia, S., Di Renzo, L., D’Orazi, G., Faggioni, A., Cirone, M., 2016. High glucose and hyperglycemic sera from type 2 diabetic patients impair DC differentiation by inducing ROS and activating Wnt/betacatenin and p38 MAPK. Biochim. Biophys. Acta 1862 (4), 805-813.

Gilardini Montani, M.S., Santarelli, R., Falcinelli, L., Gonnella, R., Granato, M., Di Renzo, L., Cuomo, L., Vitillo, M., Faggioni, A., Cirone, M., 2018a. EBV up-regulates PD-L1 on the surface of primary monocytes by increasing ROS and activating TLR signaling and STAT3. J. Leukoc. Biol. 104 (4), 821-832.

Gilardini Montani, M.S., Santarelli, R., Granato, M., Gonnella, R., Torrisi, M.R., Faggioni, A., Cirone, M., 2019b. EBV reduces autophagy, intracellular ROS and mitochondria to impair monocyte survival and differentiation. Autophagy 15 (4), 652-667.

Granato, M., Santarelli, R., Farina, A., Gonnella, R., Lotti, L.V., Faggioni, A., Cirone, M., 2014. Epstein-barr virus blocks the autophagic flux and appropriates the autophagic machinery to enhance viral replication. J. Virol. 88 (21), 12715-12726.

Granato, M., Santarelli, R., Filardi, M., Gonnella, R., Farina, A., Torrisi, M.R., Faggioni, A., Cirone, M., 2015. The activation of KSHV lytic cycle blocks autophagy in PEL cells. Autophagy 11 (11), 1978-1986.

Granato, M., Zompetta, C., Vescarelli, E., Rizzello, C., Cardi, A., Valia, S., Antonelli, G., Marchese, C., Torrisi, M.R., Faggioni, A., Cirone, M., 2016. HCV derived from sera of HCV-infected patients induces pro-fibrotic effects in human primary fibroblasts by activating GLI2. Sci. Rep. 6, 30649.

Himes, S.R., Sester, D.P., Ravasi, T., Cronau, S.L., Sasmono, T., Hume, D.A., 2006. The JNK are important for development and survival of macrophages. J. Immunol. 176 (4), 2219-2228.

Host, K.M., Jacobs, S.R., West, J.A., Zhang, Z., Costantini, L.M., Stopford, C.M., Dittmer, D.P., Damania, B., 2017. Kaposi's sarcoma-associated herpesvirus increases PD-L1 and proinflammatory cytokine expression in human monocytes. MBio 8 (5).

Klionsky, D.J., Abdelmohsen, K., Abe, A., Abedin, M.J., Abeliovich, H., Acevedo Arozena, A., et al., 2016. Guidelines for the use and interpretation of assays for monitoring autophagy (3rd edition). Autophagy 12 (1), 1-222.

Lee, J.S., Li, Q., Lee, J.Y., Lee, S.H., Jeong, J.H., Lee, H.R., Chang, H., Zhou, F.C., Gao, S.J., Liang, C., Jung, J.U., 2009. FLIP-mediated autophagy regulation in cell death control. Nat. Cell Biol. 11 (11), 1355-1362.

Liang, Q., Chang, B., Brulois, K.F., Castro, K., Min, C.K., Rodgers, M.A., Shi, M., Ge, J., Feng, P., Oh, B.H., Jung, J.U., 2013. Kaposi's sarcoma-associated herpesvirus K7 modulates Rubicon-mediated inhibition of autophagosome maturation. J. Virol. 87 (22), 12499-12503.

Lupertz, R., Chovolou, Y., Kampkotter, A., Watjen, W., Kahl, R., 2008. Catalase overexpression impairs TNF-alpha induced NF-kappaB activation and sensitizes MCF-7 cells against TNF-alpha. J. Cell. Biochem. 103 (5), 1497-1511.

Park, G.B., Kim, Y.S., Lee, H.K., Song, H., Cho, D.H., Lee, W.J., Hur, D.Y., 2010. Endoplasmic reticulum stress-mediated apoptosis of EBV-transformed B cells by cross-linking of CD70 is dependent upon generation of reactive oxygen species and activation of p38 MAPK and JNK pathway. J. Immunol. 185 (12), 7274-7284.

Purushothaman, P., Dabral, P., Gupta, N., Sarkar, R., Verma, S.C., 2016. KSHV genome replication and maintenance. Front. Microbiol. 7, 54.

Santarelli, R., Gonnella, R., Di Giovenale, G., Cuomo, L., Capobianchi, A., Granato, M., Gentile, G., Faggioni, A., Cirone, M., 2014. STAT3 activation by KSHV correlates with IL-10, IL-6 and IL-23 release and an autophagic block in dendritic cells. Sci. Rep. 4, 4241 .

Santarelli, R., Granato, M., Faggioni, A., Cirone, M., 2015. Interference with the autophagic process as a viral strategy to escape from the immune control: lesson from gamma herpesviruses. J. Immunol. Res. 2015, 546063.

Santarelli, R., Granato, M., Pentassuglia, G., Lacconi, V., Gilardini Montani, M.S., Gonnella, R., Tafani, M., Torrisi, M.R., Faggioni, A., Cirone, M., 2016. KSHV reduces autophagy in THP-1 cells and in differentiating monocytes by decreasing CAST/calpastatin and ATG5 expression. Autophagy 12 (12), 2311-2325.

Woo, C.H., Eom, Y.W., Yoo, M.H., You, H.J., Han, H.J., Song, W.K., Yoo, Y.J., Chun, J.S., Kim, J.H., 2000. Tumor necrosis factor-alpha generates reactive oxygen species via a cytosolic phospholipase A2-linked cascade. J. Biol. Chem. 275 (41), 32357-32362.

Yamamoto, T., Sakaguchi, N., Hachiya, M., Nakayama, F., Yamakawa, M., Akashi, M. 2009. Role of catalase in monocytic differentiation of U937 cells by TPA: hydrogen peroxide as a second messenger. Leukemia 23 (4), 761-769.

Zhang, Y., Choksi, S., Chen, K., Pobezinskaya, Y., Linnoila, I., Liu, Z.G., 2013. ROS play a critical role in the differentiation of alternatively activated macrophages and the occurrence of tumor-associated macrophages. Cell Res. 23 (7), 898-914.

Zhang, Y., Morgan, M.J., Chen, K., Choksi, S., Liu, Z.G., 2012. Induction of autophagy is essential for monocyte-macrophage differentiation. Blood 119 (12), 2895-2905.

Zhong, L., Shu, W., Dai, W., Gao, B., Xiong, S., 2017. Reactive oxygen species-mediated cJun NH2-Terminal kinase activation contributes to hepatitis B virus X ProteinInduced autophagy via regulation of the Beclin-1/Bcl-2 interaction. J. Virol. 91 (15).

Zukowski, P., Maciejczyk, M., Matczuk, J., Kurek, K., Waszkiel, D., Zendzian-Piotrowska, M., Zalewska, A., 2018. Effect of N-Acetylcysteine on antioxidant defense, oxidative modification, and salivary gland function in a rat model of insulin resistance. Oxid. Med. Cell. Longev. 2018, 6581970. 